\title{
The promise of Gaia and how it will influence stellar ages
}

\author{
Carla Cacciari \\ INAF, Osservatorio Astronomico di Bologna, \\ Via Ranzani 1, 40127 Bologna, Italy \\ email: carla.cacciari@oabo.inaf.it
}

\begin{abstract}
The Gaia space project, planned for launch in 2011, is one of the ESA cornerstone missions, and will provide astrometric, photometric and spectroscopic data of very high quality for about one billion stars brighter than $\mathrm{V}=20$. This will allow to reach an unprecedented level of information and knowledge on several of the most fundamental astrophysical issues, such as mapping of the Milky Way, stellar physics (classification and parameterization), Galactic kinematics and dynamics, study of the resolved stellar populations in the Local Group, distance scale and age of the Universe, dark matter distribution (potential tracers), reference frame (quasars, astrometry), planet detection, fundamental physics, Solar physics, Solar system science.

I will present a description of the instrument and its main characteristics, and discuss a few specific science cases where Gaia data promise to contribute fundamental improvement within the scope of this Symposium.
\end{abstract}

Keywords. space vehicles, astrometry, stars: distances, stars: kinematics, stars: fundamental parameters, Galaxy: stellar content, Galaxy: fundamental parameters, Galaxy: formation, Galaxy: evolution, galaxies: stellar content

\section{Introduction}

The idea of measuring the position of stars on the sky systematically and for a scientific purpose dates back to the 2nd Century BC, when the Greek astronomer Hipparchus measured about 1000 stars naked eye. This has been repeated several times during the following centuries, with steadily increasing power and accuracy, until the most recent Hipparcos satellite (1989-1993) that measured $\sim 120,000$ sources with $\sim 1$ mas accuracy and produced a Catalogue (Perryman et al. 1997) later revised by van Leeuwen (2007).

Gaia represents the natural follow up of the Hipparcos mission, with huge improvement in terms of: i) measurement accuracy, ii) limiting magnitude and hence number of observed objects, and iii) the combination of nearly simultaneous astrometric, photometric and spectroscopic observations.

Gaia is a cornerstone mission of the ESA Space Program, that will perform an allsky survey and produce accurate astrometry and photometry for about $1.5 \times 10^{9}$ objects down to a limiting magnitude of $20 \mathrm{mag}$, and additional spectroscopy for objects brighter than $\mathrm{V}=16-17$. This will allow to obtain a stereoscopic and kinematic view of the Galaxy, and to address key questions of modern astrophysics regarding the formation and evolution of the Milky Way. Such an observational effort has been compared to the mapping of the human genome for the impact that it will have in Galactic astrophysics. In addition, Gaia will provide a fundamental contribution in a much broader range of scientific areas (see Sect. 3).

More information on the Gaia mission and its science can be found in the Gaia Concept and Technology Study Report (2000), the Proceedings of the Symposium "The 3-Dimensional Universe with Gaia" (2005), and at http://www.rssd.esa.int/Gaia. 
Table 1. Comparison of Hipparcos and Gaia characteristics and performance

\begin{tabular}{lcc}
\hline & Hipparcos & Gaia \\
\hline Completeness to $\ldots$ & $\mathrm{V} \sim 9$ & $\mathrm{~V} \sim 20$ (blue) $-22(\mathrm{red})$ \\
\hline Magnitude limit & $\mathrm{V} \sim 12.4$ & $\sim 1 \mathrm{mag}$. fainter than completeness \\
\hline $\begin{array}{l}\text { N. Sources } \\
\text { Quasars } \\
\text { galaxies }\end{array}$ & $\sim 1.210^{5}$ & $\sim 1.510^{9}$ \\
& $\sim 10^{6}$ \\
& 0 & $\sim 10^{7}$ \\
\hline Astrometric accuracy & 0 & $\leqslant 7 \mu$ as at $\mathrm{V} \leqslant 10$ \\
& $\sim 1$ mas & $100($ red $)-300($ blue $) \mu$ as at $\mathrm{V}=20$ \\
\hline Photometry & 2 bands & low-res spectrophotometry, 330-1050 nm \\
\hline Spectroscopy $(\mathrm{R} \sim 11,000)$ & none & $1-10 \mathrm{~km} \mathrm{~s}{ }^{-1}$ at $\mathrm{V} \leqslant 16($ blue $)-17(\mathrm{red})$ \\
\hline Target selection & input catalogue & real-time onboard selection \\
\hline
\end{tabular}

In Table 1 Gaia and Hipparcos characteristics and performances are compared.

\section{Overview of the Gaia mission}

Gaia is scheduled for launch at the end of 2011 from Kourou by a Soyuz-Fregat launcher, that will put it in a Lissajous-type eclipse-free orbit around L2 point of the Sun-Earth system. The design lifetime is 5 years, with a possible extension of one year. The satellite will perform a continuous scanning of the sky at a rate of $60 \operatorname{arcsec~s}^{-1}$, with a precession period of the spin axis of 70 days. As a result of this scanning law, at the end of the mission the whole sky will have been observed several times, from a few tens to more than 200 depending on the position (see Fig. 1), the average value being around 80 .

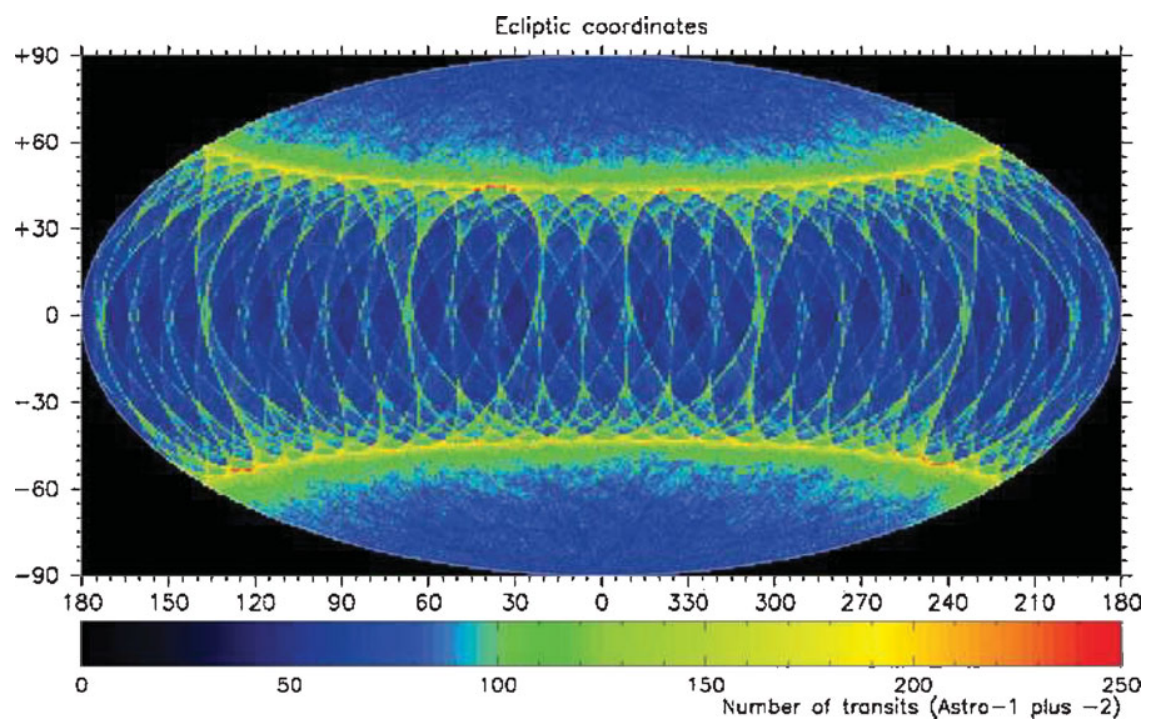

Figure 1. Dependence of the total end-of-mission number of focal plane transits on position on the sky. Shown is an all-sky equal-area Hammer projection in ecliptic coordinates. The maximum number of transits will occur in a $\sim 10$-deg wide strip around ecliptic latitudes $+/-45 \mathrm{deg}$. 


\subsection{Measurement Principle}

The mission is designed to perform global (wide field) astrometry as opposed to local (narrow field) astrometry. In local astrometry, the star position can only be measured with respect to a neighbouring star in the same field. Even with an accurate instrument, the propagation of errors is prohibitive when making a sky survey. The principle of global astrometry is to link stars with large angular distances in a network where each star is connected to a large number of other stars in every direction.

Global astrometry requires the simultaneous observation of two fields of view in which the star positions are measured and compared. Therefore, the payload will provide two lines of sight, obtained with two separate telescopes. Then, like Hipparcos, the two images will be combined, slightly spaced, on a unique focal plane assembly. Objects are matched in successive scans, attitude and calibrations are updated, and object positions are solved and fed back into the system. The procedure is iterated as more scans are added (Global Iterative Solution). In this way the system is self-calibrating by the use of isolated non variable point sources that will form a sufficiently large body of reference objects for most calibration purposes, including the definition of the celestial frame. Extragalactic objects (e.g. QSOs) will be used to attach this to the International Celestial Reference Frame.

\subsection{Instruments and data products}

The payload consists of a toroidal structure (optical bench) holding two primary mirrors whose viewing directions are separated by $106.5 \mathrm{deg}$ (the Basic Angle). These two fields of view get superposed and combined on the same focal plane, that contains:

- the Sky Mapper: an array of $2 \times 7$ CCDs for on-board star detection and selection;

- the Astrometric Field (AF): an array of $9 \times 7$ CCDs for astrometric measurements and integrated white-light photometry;

- the Blue (BP) and Red (RP) Photometers: two columns of 7 CCDs each for prism spectrophotometry in the 330-680 nm wavelength range with dispersion 3-29 nm/px, and in the $640-1050 \mathrm{~nm}$ wavelength range with dispersion 7-15 nm/px, respectively;

- the Radial Velocity Spectrometer (RVS): an array of $3 \times 4$ CCDs for slitless spectroscopy (through grating and afocal field corrector) at the Ca II triplet (847-874 nm) with resolution $\mathrm{R} \sim 11,000$.

Therefore the data produced by Gaia will be of three types:

- Astrometry (parallaxes, proper motions);

- Photometry, both integrated (such as the white-light $G$-band from the Astrometric Field and the $G_{B P}$ and $G_{R P}$ from the blue and red photometers) and low-dispersion $\mathrm{BP}$ and RP spectra. We show in Figure 2 examples of simulated BP and RP spectra as will be observed by Gaia, for main sequence stars from $\mathrm{O} 5$ to $\mathrm{M} 6$, at $\mathrm{G}=15$ and zero reddening (Straižys et al. 2006).

- Spectroscopy (radial velocities; rotation, chemistry for the brighter sources).

\subsection{Performances}

\subsubsection{Astrometry}

Astrometric errors are dominated by photon statistics. The expected accuracies of parallax measures as a function of $\mathrm{V}$ are listed in Table 1, and are also reported as a function of $\mathrm{M}_{V}$ (i.e. distance) in Table 2 (left part), along with the corresponding number of objects that can be observed by Gaia. We consider $\mathrm{V}=7 \mathrm{mag}$ as the bright magnitude limit for astrometric observations, as saturation sets in at about $\mathrm{V}=6$ mag.

Accuracy on proper motions is about $20 \%$ better than on parallaxes. 

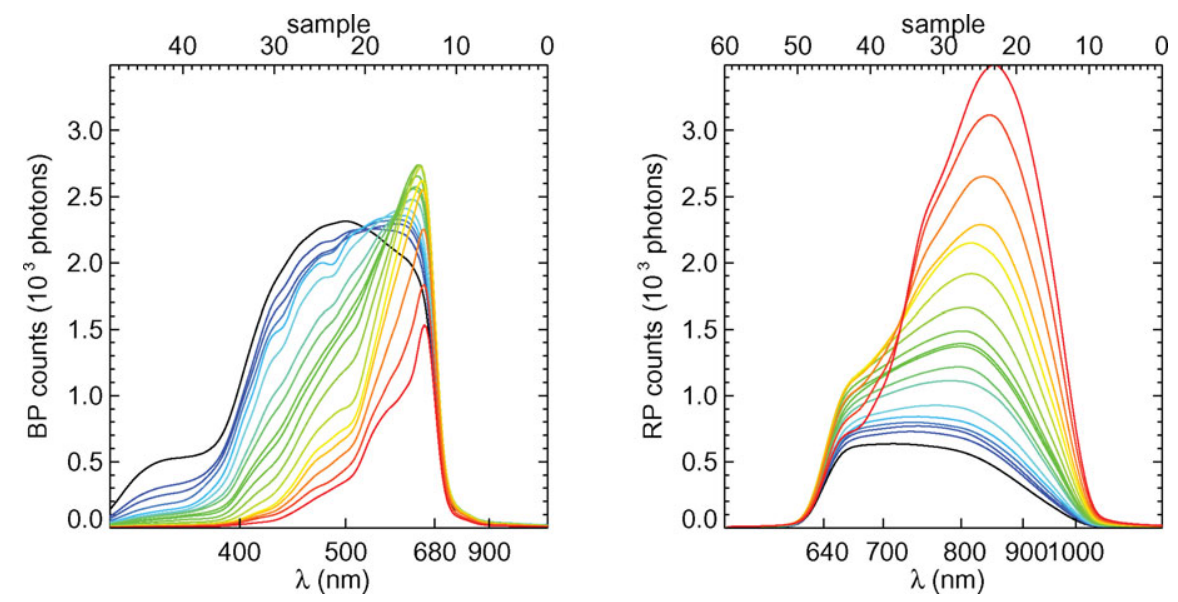

Figure 2. Examples of simulated $\mathrm{BP}$ and RP prism spectra for main sequence stars from $\mathrm{O} 5$ to $\mathrm{M} 6$, at $\mathrm{G}=15$ and $\mathrm{A}_{V}=0$ (Straižys et al. 2006).

Table 2. Accuracy of astrometric and photometric data. Left: parallax accuracy (and number of objects) as a function of $\mathrm{M}_{V}$ and $\mathrm{V}$ (and hence distance). Right: accuracy of internally calibrated integrated photometry as a function of $G$ mag.

\begin{tabular}{|c|c|c|c|c|c|c|c|c|c|c|c|}
\hline $\begin{array}{c}\sigma(\pi) / \pi \\
\text { N. of objects }\end{array}$ & $\mathrm{M}_{V}$ & -5 & 0 & 5 & 10 & 15 & $\begin{array}{c}G \\
\operatorname{mag}\end{array}$ & 13 & 15 & 17 & 20 \\
\hline $\begin{array}{c}0.1-0.3 \% \\
\sim 10^{5}\end{array}$ & $\begin{array}{c}\mathrm{V} \\
\mathrm{D}(\mathrm{pc})\end{array}$ & & $\begin{array}{c}7 \\
250\end{array}$ & $\begin{array}{c}12 \\
250\end{array}$ & $\begin{array}{c}15 \\
100\end{array}$ & $\begin{array}{l}17 \\
25\end{array}$ & $\begin{array}{c}\sigma G \\
\mathrm{mmag}\end{array}$ & 0.1 & 0.2 & 0.8 & 2.5 \\
\hline $\begin{array}{l}1-3 \% \\
\sim 10^{7}\end{array}$ & $\begin{array}{c}\mathrm{V} \\
\mathrm{D}(\mathrm{pc})\end{array}$ & $\begin{array}{c}7 \\
2500\end{array}$ & $\begin{array}{c}12 \\
2500\end{array}$ & $\begin{array}{c}15 \\
1000\end{array}$ & $\begin{array}{c}17 \\
250\end{array}$ & $\begin{array}{c}20 \\
100\end{array}$ & $\begin{array}{l}\sigma G_{B P} \\
\mathrm{mmag}\end{array}$ & 0.3 & 0.8 & 6.5 & 37.8 \\
\hline $\begin{array}{c}10-30 \% \\
\geqslant 10^{8}\end{array}$ & $\begin{array}{c}\mathrm{V} \\
\mathrm{D}(\mathrm{pc})\end{array}$ & $\begin{array}{c}12 \\
25000\end{array}$ & $\begin{array}{c}15 \\
10000\end{array}$ & $\begin{array}{c}17 \\
2500\end{array}$ & $\begin{array}{c}20 \\
1000\end{array}$ & & $\begin{array}{l}\sigma G_{R P} \\
\mathrm{mmag}\end{array}$ & 0.3 & 0.7 & 6.1 & 35.2 \\
\hline
\end{tabular}

We note that comparable astrometric accuracy is (will be) obtained from other (present) future space and ground-based facilities, but on pencil-beam areas of the sky.

\subsubsection{Photometry}

We show in Table 2 (right part) a summary of the accuracy that Gaia is expected to achieve for the internally calibrated integrated photometry in white light ( $G$-band) and in the $G_{B P}$ and $G_{R P}$ bands, as a function of $G$ mag. The quoted values are predicted end-of-mission (80 transits) mean values and include Poisson, background and readout noise. A systematic $\sim 1 \mathrm{mmag}$ calibration error should be considered in addition (Jordi et al. 2007). The accuracy per pixel of the BP/RP spectrophotometric data will be at least one order of magnitude worse, depending on the shape of the spectral energy distribution.

The external (absolute) flux calibration of the Gaia photometric system will be derived from constraining observations of spectrofotometric standard stars (SPSS). To this purpose a grid of SPSS is being built, based on CALSPEC stars and incremented by additional stars to cover adequately the widest possible spectral type range. An observing campaign has been started to ensure accurate and homogeneous data for a suitably large number of SPSS. The accuracy of the absolute calibrated data is expected to be $\sim 1 \%$ 
to a few percent, depending on the number and spectral type of the SPSS and on the accuracy of their SEDs, and may vary with wavelength across the BP/RP spectra.

¿From the BP/RP spectral energy distributions it will be possible to estimate astrophysical parameters using pattern recognition techniques (Bailer-Jones 2008). For example, one expects to obtain (r.m.s. are internal uncertainties at $\mathrm{V}=15$ ):

- $\mathrm{T}_{\text {eff }}$ to $1-5 \%$ for a wide range of $\mathrm{T}_{e f f}$;

- $\log g$ to 0.1-0.4 dex, $<0.1$ dex for hot stars (SpT $\leqslant \mathrm{A})$;

- $[\mathrm{Fe} / \mathrm{H}]$ to $<0.2$ dex for cool stars $(\mathrm{SpT}>\mathrm{F})$ down to $[\mathrm{Fe} / \mathrm{H}]=-2.0$ dex;

- $\mathrm{A}_{V}$ to 0.05-0.1 mag for hot stars;

thus providing a complete characterisation of stellar populations.

\subsubsection{Spectroscopy}

The RVS provides the third component of the space velocity of each red (blue) source down to about 17 th (16th) magnitude.

Radial velocities are the main product of the RVS, with typical accuracies of 1 to 10 $\mathrm{km} \mathrm{s}^{-1}$ down to the limiting magnitude. For brighter sources $(<14 \mathrm{mag})$ the RVS spectra will provide information also on rotation and chemistry, and will allow to obtain more detailed and accurate astrophysical parameters than using the prism BP/RP spectra alone.

\subsection{Science data processing: the DPAC}

All aspects of the Gaia mission are charge and responsibility of ESA, except the processing and analysis of the science data that are assigned to the European astronomical community. To this purpose the community has formed the Data Processing and Analysis Consortium (DPAC), that collects the contribution of nearly 400 scientists from 24 Institutes of ESA member States, and is structured in 9 Coordination Units dealing with all aspects of the data processing. In addition, a number of Data Processing Centers (DPCs) are dedicated to the data handling and processing of specific parts of the pipeline, namely: ESAC (Spain), CNES (France) and the DPCs in Barcelona, Torino, Toulouse, Cambridge and Geneva.

A final data Catalogue will be produced around 2019-2020, containing the end-ofmission measurements for the complete sample of objects down to $\mathrm{V}=20 \mathrm{mag}$. Intermediate catalogues might be released before the end of the mission, as appropriate. Science alerts data are released immediately.

No proprietary data rights are implemented.

\section{Science with Gaia}

The primary goal of the Gaia mission is to obtain data which allow for studying the structure, composition, formation and evolution of the Galaxy. The detailed knowledge of the Galaxy will provide a firm base for the analysis of other galaxies for which this level of accuracy cannot be achieved through direct observations. However, a large number of objects external to the Galaxy will be reached by the Gaia instruments, yielding results of no less interest and importance.

\subsection{Science products}

In the Galaxy: Gaia will provide a complete census of all stellar populations down to $\overline{20 t h}$ magnitude. Based on the Besançon Galaxy model (Robin et al. 2003, 2004) Gaia is expected to measure about $9 \times 10^{8}$ stars belonging to the Disk, $4.3 \times 10^{8}$ Thick Disk stars, $2.1 \times 10^{7}$ Spheroid stars and $1.7 \times 10^{8}$ Bulge stars. Binaries, variable stars and rare 
stellar types (fast evolutionary phases) will be well sampled, as well as special objects such as Solar System bodies $\left(\sim 10^{5}\right)$, extra-solar planets $\left(\sim 2 \times 10^{4}\right)$, WDs $\left(\sim 2 \times 10^{5}\right)$, BDs $\left(\sim 5 \times 10^{4}\right)$.

One billion stars in 5-D (6-D if the radial velocity is available, and up to 9-D if the astrophysical parameters are know as well) will allow to derive the spatial and dynamical structure of the Milky Way, its formation and chemical history (e.g. by detecting evidence of accretion/merging events), and the star formation history throughout the Galaxy. The huge and accurate database will provide a powerful testbench for stellar structure and evolution models. The possibility to obtain clean Colour-Magnitude (and hence HR) diagrams throughout the Galaxy will lead to accurate mass and luminosity functions, as well as complete characterisation and dating of all spectral types and Galactic stellar populations. The distribution and rate of microlensing events will allow to map the dark matter distribution. The cosmic distance scale will get a definitive and robust definition (zero-point) thanks to the very accurate distance (i.e. luminosity calibration) of the primary standard candles, RR Lyraes and Cepheids.

Outside the Galaxy: the brightest stars in nearby (LG) galaxies will be observed by Gaia, as well as SNe and burst sources $\left(\sim 2 \times 10^{4}\right)$, distant galaxies $\left(\sim 10^{7}\right)$, QSOs $\left(\sim 10^{6}\right)$, gravitational lensing events $\left(\leqslant 10^{2}\right.$ photometric, a few $10^{2}$ astrometric $)$.

Fundamental physics and general relativity will also benefit from Gaia observations: as an example, the parameter $\gamma$, representing the deviation from Newtonian theory of the gravitational light bending, will be measured to $\sim 5 \times 10^{-7}$ as compared to the present accuracy of $10^{-4}-10^{-5}$.

\subsection{The impact of Gaia on stellar ages: a few examples}

\subsubsection{Globular clusters}

Globular clusters (GCs) are among the densest fields in the sky, and considering that the maximum density that Gaia can handle is $\sim 0.25 \mathrm{star}_{\operatorname{arcsec}}^{-2}$ not all of the $150 \mathrm{GCs}$ known in the Galaxy (Harris 1996) will be completely observable right to the centre. Simulations with King models and concentration parameter $c=0.5$ to 2.5 have shown that $30 \%$ of them can be fully observed, most of the remaining ones can be observed at radial distances $\mathrm{r}>1$ arcmin, and only 5 will be observable at $\mathrm{r}>3$ arcmin. Therefore, for more than $100 \mathrm{GCs}$ it will be possible to observe from $10^{3}$ up to $10^{6}$ stars each.

The availability of parallaxes and proper motions, as well as radial velocities for $\mathrm{V}$ $<16-17$, allows to assess the membership and hence to derive clean CM diagrams. At the limiting magnitude $\mathrm{V}=20$, corresponding to $\geqslant 1$ mag below the main-sequence turnoff for the 30 GCs closer than $10 \mathrm{kpc}$, the accuracy on proper motions is expected to be $\sim 0.08$ mas (for red stars) to $\sim 0.25$ mas (for blue stars).

For comparison, we note that King et al. (1998), in their seminal work on NGC 6397, were able to perform a very good cleaning of the main-sequence using proper motions of accuracy $\sim 10$ mas obtained from WFPC2-WFC data over a 32 month time baseline. The most recent achievement by Anderson \& King (2006) is $~ 0.5$ mas astrometric accuracy from ACS/WFC data on well exposed images. This accuracy is indeed getting close to Gaia's, but on a field of view of only about $200 \times 200 \operatorname{arcsec}^{2}$.

Clean CMD are the essential tool to derive the absolute (and relative) age of a GC, as we discuss in more detail in the following section using M3 as an example.

\subsubsection{The age of $M 3$}

At a distance of about $10 \mathrm{kpc}$, the brightest features of M3 CM diagram, namely the upper RGB and the HB, are at $\mathrm{V} \sim 12.5$ to 16 mag. Therefore these stars will be observed with individual accuracy $\sigma(\pi) / \pi \sim 7-30 \%$. By averaging the results from 1000 such stars 
distributed according to the RGB luminosity function (Ferraro et al. 1997), the distance to M3 can be known to about $0.5 \%$ or better.

The most classical clock provided by stellar evolution theory for dating Population II stars is the luminosity of turn-off stars $\mathrm{M}_{V}$ (TO). This has been parameterised by Renzini (1993) as:

$$
\operatorname{Logt}_{9} \propto 0.37 M_{V}(T O)-0.43 Y-0.13[\mathrm{Fe} / \mathrm{H}]
$$

$\mathrm{M}_{V}$ (TO) is sensitive to input physics and assumptions that affect the size and energy production of the radiative core. Comparison of the various most recent $\mathrm{M}_{V}(\mathrm{TO})$ vs. age relations shows that an intrinsic - and hence systematic - theoretical error in the age determination may be present. We refer to the presentation by B. Chaboyer (this conference) for more details on the intrinsic/systematic errors of theoretical models.

In addition to this, errors on the observable parameters entering the $\mathrm{M}_{V}$ (TO)-age relation must be considered. By assuming typical values currently obtained for these errors, the error budget can be summarised as:

- $0.85 \sigma\left(\mathrm{V}_{T O}\right)$ : error associated to the photometric determination of the TO. Extremely accurate and well defined main sequences can presently be obtained with instruments such as the HST and other large ground-based facilities, however the isochrones are nearly vertical at the $\mathrm{TO}$, and $\mathrm{V}_{T O}$ is rather difficult to define. We assume $\sigma\left(\mathrm{V}_{T O}\right) \sim 0.10 \mathrm{mag}$;

- $0.85 \sigma(\mathrm{mod})$ : error associated to the distance determination, we assume $\sim 0.10 \mathrm{mag}$;

- $0.85 \sigma\left(\mathrm{A}_{V}\right)$ : error associated to the extinction determination, we assume $\sim 0.06 \mathrm{mag}$;

- $0.99 \sigma(\mathrm{Y})$ : error associated to the Helium abundance determination, we assume $\geqslant 0.02$ dex;

- $0.30 \sigma[\mathrm{M} / \mathrm{H}]$ : error associated to the chemical abundance determination, we assume $\sim 0.10$ dex.

The final accuracy on age determinations is $\sim 13 \%$, as also estimated by A. Sarajedini (this conference). In a few particular cases and with especially accurate data and analysis, the accuracy on age determination has been claimed to be as low as $\leqslant 10 \%$ (Gratton et al. 2003). However, before further improvement can be achieved systematic errors need to be solved, for example on chemical abundance determination (by defining the calibrating Solar mixture and metallicity scale), on helium abundance determination (which is confused by the possible presence of multiple populations), on the definition of a homogeneous reddening scale.

Gaia will do its share to improve absolute age by acting on most of the above items: i) clean CMDs and very accurate photometry at the level of the turn-off will allow to obtain a more precise definition and estimate of the observed $\mathrm{V}_{T O}$. Accuracies of $\sim 0.01-0.02$ mag can be foreseen, and are within reach also of the best present and future observing facilities.

ii) The reddening will be monitored by Gaia for each object as part of the astrophysical parameter determination and may not be very accurate individually, but the statistical use of all cluster stars could lead to a rather accurate mean estimate. To be conservative, we assume a factor two improvement in the accuracy of the reddening values.

iii) Similarly, $[\mathrm{Fe} / \mathrm{H}]$ will be estimated as part of the astrophysical parameter determination, and the mean of hundreds/thousands stars will carry rather low internal errors. iv) The most important contribution, however, will be on the distance determination, by greatly reducing the error on distance (e.g. by a factor 10 at $10 \mathrm{kpc}$ ).

Altogether, we expect that absolute ages can be known to $\sim 5 \%$ or better. 


\subsubsection{Open clusters}

Open clusters (OCs) are much looser than GCs and will be completely observable to the centre by Gaia. Therefore, the same type of analysis described for GCs in Sections 3.2.1 and 3.2.2 can be applied to the entire stellar population for each and all of the presently known OCs. In addition, Gaia data may well be able to identify new (faint/loose) clusters.

With Hipparcos, firm results were obtained only on 7 OCs, whereas the Pleiades still represent a controversial case.

The Pleiades have been studied extensively in the last decade, and very similar parallax values have been found by various authors: $\pi=7.59 \pm 0.14$ mas from MS fitting (Pinsonneault et al. 1998), $\pi=7.69$ mas from various methods (Kharchenko et al. 2005), $\pi=7.49 \pm 0.07$ mas from HST-FGS parallaxes of three stars in the inner halo (Soderblom et al. 2005). However, from the new reduction of Hipparcos data van Leeuwen (2007) finds $\pi=8.18 \pm 0.13$ mas. This discrepancy of about $8 \%$ in the distance determination will be resolved unambiguously by Gaia. Since all the stars of the Pleiades are brighter than $\mathrm{V}=15$, they will have individual parallaxes determined to better than $0.1-0.2 \%$, and the distance and internal stellar distribution will be derived with extremely high precision.

\subsubsection{The distance scale: local calibrators}

\section{- RR Lyraes}

RR Lyrae variable stars are the most traditional standard candles, as their absolute magnitude can be expressed to a first approximation as $M_{V}=\alpha+\beta[\mathrm{Fe} / \mathrm{H}]$, with $\beta \sim 0.2$. However, the zero-point $\alpha$ of this relation is determined to somewhat lower accuracy than the slope $\beta$.

Hipparcos measured parallaxes for $126 \mathrm{RR}$ Lyrae stars with $\langle\mathrm{V}\rangle=10$ to $12.5 \mathrm{mag}$ (750-2500 pc, Fernley et al. 1998), but only one star, RR Lyr itself, had a parallax measured to better than $20 \%, \pi=3.46 \pm 0.64$ mas (van Leeuwen 2007). However, the parallax measured by Benedict et al. (2002) using HST data, $\pi=3.82 \pm 0.20$ mas, leads to a shorter distance modulus by $\sim 0.2 \mathrm{mag}$. This discrepancy is far too large and definitely not acceptable for what is supposed to be the basic luminosity/distance calibrator and the first step in the cosmic distance scale.

Gaia will obtain the parallax of RR Lyr to $<0.1 \%$ and the trigonometric distances to all the field RR Lyraes within $3 \mathrm{kpc}$ with individual accuracy $\sigma(\pi) / \pi<3 \%$ (better than $30 \%$ for most galactic RR Lyraes). This will allow to calibrate the $M_{V}-[\mathrm{Fe} / \mathrm{H}]$ relation with very high accuracy, for application to all stellar systems where a good estimate of the RR Lyrae metallicity and mean $\mathrm{V}$ magnitude is possible.

\section{- Cepheids}

Cepheids, along with RR Lyrae stars, form the cornerstone of the extragalactic distance scale. Classical (Pop I) Cepheids are several magnitudes brighter than RR Lyraes, and can be observed in many spiral and irregular galaxies as far as $25 \mathrm{Mpc}$ (thus reaching the Fornax and Virgo clusters) with the use of the HST and other large ground-based or space telescopes. The Hipparcos data provided the first opportunity to calibrate independently the critical parameters in the Period-Luminosity-Colour (PLC) relation for classical Cepheids in the Galaxy. Hipparcos measured parallaxes for about 250 Cepheids, $\sim 100$ of which with parallax accuracies of 1 mas or less. With the use of these data and additional HST parallax measures for 10 of these stars, van Leeuwen et al. (2007) derived a new calibration of the PLC relation leading to a distance modulus for the LMC of $18.48 \pm 0.03 \mathrm{mag}$ (no metallicity correction), and hence $\mathrm{H}_{0}=70 \pm 5 \mathrm{~km} \mathrm{~s}^{-1} \mathrm{Mpc}^{-1}$. This is certainly an excellent result, but is still affected by uncertainties due to the various parameters involved in the definition of the calibration itself. 
Gaia is expected to measure distances to $<4 \%$ for all galactic Cepheids $(<1 \%$ up to 3 $\mathrm{kpc}$ ), therefore will provide a definitive resolution of the controversy about the zero-point of the PLC relation, as well as about the dependence on period, colour and metallicity.

Cepheid parallaxes can also be measured by Gaia in extragalactic systems such as the Sagittarius dwarf galaxy with $\sigma(\pi) /(\pi)<10 \%$, and the Magellanic Clouds with $\sigma(\pi) /(\pi)$ $\leqslant 50 \%$ for all stars with period longer than $\sim 10$ days $\left(M_{V} \leqslant-4.2 \mathrm{mag}\right)$.

This will allow to reach a few fundamental goals:

i) define a very accurate PLC relation, including the possible dependence on metallicity; ii) establish the distance to the LMC on a completely trigonometric basis, and improve its accuracy with the additional help of the Galactic calibration relation;

iii) establish the universality of the PLC relation, namely its applicability to all galaxies and hence the possibility to derive $\mathrm{H}_{0}$ and the age of the Universe.

\section{- Metal-Poor Subdwarfs}

Within the context of distance determination, metal-poor subdwarfs are very important as they constitute the reference frame for GC main sequence stars of similar metallicity. The availability of the high precision Hipparcos parallaxes prompted numerous determinations of distances to several Galactic GCs using this Main Sequence Fitting method. We refer the reader to e.g. Gratton et al. (2003) for a detailed description and review.

However, Hipparcos provided precise enough parallaxes only for $\sim 30$ metal-poor subdwarfs with $M_{V} \sim 5.5$ to $7.5 \mathrm{mag}$ (i.e. 2-4 mag below the TO). Since the required astrometric accuracy could only be obtained within limiting magnitude $\mathrm{V} \sim 10$, this allowed to sample a rather small volume of the local neighborhood within $30-80 \mathrm{pc}$ (and hence the small number of metal-poor stars).

Gaia's limiting magnitude to $\mathrm{V} \sim 15$ will allow to measure metal-poor subdwarfs in the same range of absolute magnitude as far as $\sim 800 \mathrm{pc}$ with astrometric accuracy better than $\sim 3 \%$. Several thousands are expected, thus providing a much better statistics and finer sampling in metallicity for a more accurate fitting to any given GC main sequence.

\section{Acknowledgements}

This overview of the Gaia project borrows freely from previous scientific and technical publications, and from the information available on the Gaia website. The effort of the many people involved in the Gaia project is implicit in this synthesis.

This work was done under the financial support of ASI grant I/016/07/0 and PRININAF grant CRA1.06.10.04. The author gratefully acknowledges the support of a IAU travel grant.

\section{References}

Anderson, J. \& King, I. R. 2006, STScI Instrument Science Report ACS 2006-01

Bailer-Jones, C. A. L. 2008, Gaia-C8-TN-MPIA-CBJ-040 in Gaia Livelink

Benedict, G. F., McArthur, B. E., Fredrick, L. W. et al. 2002, AJ, 123, 473

Gaia Concept and Technology Study Report 2000, ESA-SCI(2000)4

Fernley, J., Barnes, T. G., Skillen, I., Hawley, S. L., Hanley, C. J., Evans, D. W., Solano, E., \& Garrido, R. 1998, A\&A, 330, 515

Ferraro, F. R., Carretta, E., Corsi, C. E. Fusi Pecci, F., Cacciari, C., Buonanno, R., Paltrinieri, B., \& Hamilton, D. 1997, A\&A, 320, 757

Gratton, R. G., Bragaglia, A., Carretta, E., Clementini, G., Desidera, S., Grundhal, F., \& Lucatello, S. 2003, A\&BA, 408, 529

Harris, W. E. 1996, AJ, 112, 1487 (updates in http://www.physics.mcmaster.ca/Globular.html) 
Jordi, C., Fabricius, C., Figueras, F., Voss, H., \& Carrasco, J. M. 2007, Gaia-C5-TN-UB-CJ-042 in Gaia Livelink

Kharchenko, N. V., Piskuniv, A. E., Röser, S., Schilbach, E., \& Scholz, R. D. 2005, A 6 A, 440, 403

King, I., Anderson, J., Cool, A., \& Piotto, G. 1998, ApJ, 492, L37

van Leeuwen, F. 2007, Hipparcos, the New Reduction of the Raw Data, Springer, ASSL 350

van Leeuwen, F., Feast, M. W., Whitelock, P. A., \& Laney, C. D. 2007, MNRAS, 379, 723

Perryman, M. A. C, Bernacca, P. L., \& SOC 1997, ESA SP-402

Pinsonneault, M. H., Stauffer, J., Soderblom, D. R., King, J. R., \& Hanson, R. B. 1998, ApJ, 504,170

Renzini, A. 1993, Ann. NY Acad. Sci., 688, 124

Robin, A. C., Reylé, C., Derrière, S., \& Picaud, S. 2003, A\&A, 409, 523

Robin, A. C., Reylé, C., Derrière, S., \& Picaud, S. 2004, A\&A, 416, 157

Soderblom, D. R., Nelan, E., Benedict, G. F., McArthur, B., Ramirez, I., Spiesman, W., \& Jones, B. F. 2005, AJ, 129, 1616

Straižys, V., Lazauskaite, R., Brown, A. G. A., \& Zdanavičius, K. 2006, Gaia-C8-TN-ITPAVS-001 in Gaia Livelink

Symposium "The 3-Dimensional Universe with Gaia" 2005, ESA-SP-576

\section{Discussion}

C. Corbally: What relation will Gaia have with Earth- based surveys such as PanSTARRS and LSST? Will there be collaboration and/or overlap between the data processing and analysis teams?

C. CACCIARI: The Gaia Data Processing and Analysis Consortium is totally European and there is no official collaboration or overlap with other teams. But on some specific issues there is indeed collaboration with "external" advisors, for example on the selection and observation of standard stars for the absolute calibration of the spectrophotometric system.

M. RobBerto: How much degradation do you expect from radiation damage to the CCD detectors?

C. CACCIARI: This is presently being tested by Astrium and closely monitored by a dedicated DPAC team. Solutions have been proposed (e.g., charge injection), but a definitive answer will be available only when the test will be completed, in April 2009. 